P. N. Lebedev Physical Institute

Preprint FIAN/TD/4-94

I. E. Tamm Theory Department

April 1994

\title{
ON THE MULTILEVEL FIELD-ANTIFIELD FORMALISM WITH THE MOST GENERAL LAGRANGIAN HYPERGAUGES
}

\author{
I. A. Batalin and I. V. Tyutin \\ Department of Theoretical Physics \\ P. N. Lebedev Physical Institute \\ Leninsky prospect, 53, 117 924, Moscow, Russia ${ }^{\dagger}$
}

\begin{abstract}
The multilevel field-antifield formalism is constructed in a geometrically covariant way without imposing the unimodularity conditions on the hypergauge functions. Thus the previously given version $[1,2]$ is extended to cover the most general case of Lagrangian surface bases. It is shown that the extra measure factors, required to enter the gauge-independent functional integrals, can be included naturally into the multilevel scheme by modifying the boundary conditions to the quantum master equation.
\end{abstract}

† E-mail address: batalin@fian.free.net,tyutin@fian.free.net 


\section{Introduction}

In Refs. $[1,2]$ we have suggested a geometrically covariant multilevel generalization of the field-antifield BV formalism [3-5]. As is well known, the field-antifield formalism is nothing else but the universal hypergauge theory whose action is determined by the quantum master equation, and the corresponding hypergauge generators are nilpotent.

To eliminate the characteristic degeneracy of the universal BV theory, one needs the hypergauge conditions singling out a Lagrangian surface in the field-antifield phase space. By definition, the surface $G_{a}=0$ is called the Lagrangian one if the antibracket involution relations $\left(G_{a}, G_{b}\right)=G_{c} U_{a b}^{c}$ are satisfied. However, in Refs. [1,2] we have imposed stronger restriction on the hypergauge functions $G_{a}$ by requiring the unimodularity conditions $\Delta G_{a}-U_{b a}^{b}(-1)^{\varepsilon_{b}}=G_{b} V_{a}^{b}$ to be satisfied too, where $\Delta$ is the well known nilpotent operator entering the quantum master equation $\Delta \exp \left(\frac{\imath}{\hbar} W\right)=0$. As it has been explained in Refs. [1,2], the unimodularity conditions do not restrict the arbitrariness of an intrinsic gauge fermion encoded by means of the functions $G_{a}$. Nevertheless, these extra conditions restrict rather strongly the possibilities to change the Lagrangian surface basis by making linear transformations of the form $G_{a} \rightarrow G_{b} \Lambda_{a}^{b}$. Being the unimodularity conditions fulfilled, the Jacobian of the $\delta$-functions $\delta(G)$ is constant, and the admitted generators $\delta \Lambda_{b}^{a}$ of the infinitesimal basis transformations, $\Lambda_{b}^{a}=\delta_{b}^{a}+\delta \Lambda_{b}^{a}$, should be supertraceless : $(-1)^{\varepsilon_{a}} \delta \Lambda_{a}^{a}=0$.

In the present paper we cancel the unimodularity conditions and thereby extend the construction of Refs. [1,2] to cover the most general case of the Lagrangian surface bases. Thus the antibracket involution relations and usual admissibility conditions are the only restrictions imposed on the hypergauge functions.

In order to provide for a gauge independence to the functional integral, we insert the extra measure factor into the integrand, and then convert former unimodularity conditions into the equations determining this extra measure to absorb the transformation of the hypergauge $\delta$-function Jacobian.

As a result, it will be shown that all the extra measure factors can be included naturally into the multilevel scheme by modifying the boundary conditions to the quantum master equation.

As usual, we denote by $\varepsilon(A)$ the Grassmann parity of a quantity $A$, and $\operatorname{Ber} K$ stands for the Berezinian (superdeterminant) of a supermatrix $K$.

\section{A Modified Version to the First Level Formalism}

Let $\Gamma^{A}, A=1, \ldots, 2 N, \varepsilon\left(\Gamma^{A}\right) \equiv \varepsilon_{A}$, be a total set of field-antifield variables coordinatizing the original phase space locally.

We define the antisymplectic differential $\Delta$ to be a general second-order fermionic oper- 
ator without the derivativeless term,

$$
\Delta=\frac{1}{2}(-1)^{\varepsilon_{A}} M^{-1} \partial_{A} M E^{A B} \partial_{B}
$$

required to satisfy the nilpotency condition, $\Delta^{2}=0$, so that $E^{A B}(\Gamma)$ appears to be antisymplectic metric satisfying the Jacobi identity and thus yielding the antibracket operation

$$
(F, G) \equiv F \overleftarrow{\partial_{A}} E^{A B} \overrightarrow{\partial_{B}} G
$$

Let us remind here the corresponding differentiation formulae for the antibracket (2.2) and ordinary product $F G$ :

$$
\begin{gathered}
\Delta(F, G)=(\Delta F, G)+(F, \Delta G)(-1)^{\varepsilon(F)+1}, \\
\Delta(F G)=(\Delta F) G+(F, G)(-1)^{\varepsilon(F)}+F(\Delta G)(-1)^{\varepsilon(F) .}
\end{gathered}
$$

A modified version to the first level functional integral is defined as follows :

$$
Z=\int \exp \left\{\frac{i}{\hbar}\left[W(\Gamma ; \hbar)+G_{a}(\Gamma ; \hbar) \pi^{a}\right]-H(\Gamma ; \hbar)\right\} d \mu,
$$

where

$$
d \mu=M d \Gamma d \pi
$$

is the integration measure, the action $W(\Gamma ; \hbar)$ satisfies the quantum master equation

$$
\Delta \exp \left\{\frac{i}{\hbar} W(\Gamma ; \hbar)\right\}=0
$$

$\pi^{a}, a=1, \ldots, N, \varepsilon\left(\pi^{a}\right) \equiv \varepsilon_{a}$, are the Lagrangian multipliers introducing the hypergauge functions $G_{a}$ that satisfy the general involution relations

$$
\left(G_{a}, G_{b}\right)=G_{c} U_{a b}^{c}
$$

with some structure coefficients $U_{a b}^{c}(\Gamma ; \hbar)$, the function $H(\Gamma ; \hbar)$ satisfies the equations

$$
\left(H, G_{a}\right)=\Delta G_{a}-U_{b a}^{b}(-1)^{\varepsilon_{b}}-G_{b} V_{a}^{b}
$$

with some structure coefficients $V_{a}^{b}(\Gamma ; \hbar)$. It follows from $(2.9)$ that

$$
\Delta H-\frac{1}{2}(H, H)+V_{a}^{a}=G_{a} \tilde{G}^{a}
$$

with $\tilde{G}^{a}(\Gamma ; \hbar)$ to be some functions of the original phase variables.

As compared with the previously given version $[1,2]$ of the first level formalism, the modified functional integral (2.5) is the most generalization in what concerns the choice of 
hypergauge functions $G_{a}$. The only restriction, imposed on $G_{a}$ in the present version, is that these functions should satisfy the general involution relations (2.8). That means that the hypergauge functions $G_{a}$ describe, from the purely geometric viewpoint, the most general basis of the Lagrangian surface $G_{a}=0$.

In order to provide for a gauge independence to the modified functional integral (2.5), one should insert the extra measure factor $\exp (-H)$ into the integrand of $(2.5)$, and then convert former unimodularity conditions into the equations (2.9) determining the function $H$ under the only conditions (2.8) imposed on $G_{a}$. It is evident that the modified version (2.5) coincides with the previously given one if the r.h.s. of (2.9) vanishes.

Now, let us consider the gauge independence in more details. First of, all we observe that the integrand of (2.5) is invariant under the generalized BRST-type transformations:

$$
\begin{gathered}
\delta \Gamma^{A}=\left(\Gamma^{A},-W+G_{a} \pi^{a}+\imath \hbar H\right) \mu, \\
\delta \pi^{a}=\left(-U_{b c}^{a} \pi^{c} \pi^{b}(-1)^{\varepsilon_{b}}+2 i \hbar V_{b}^{a} \pi^{b}+2(i \hbar)^{2} \tilde{G}^{a}\right) \mu,
\end{gathered}
$$

where $\mu=$ const, $\varepsilon(\mu)=1$.

Choosing the parameter $\mu$ to be an arbitrary function

$$
\mu=\frac{i}{2 \hbar} \delta X(\Gamma)
$$

and making the additional variations

$$
\delta \Gamma^{A}=\frac{1}{2}\left(\Gamma^{A}, \delta X\right), \quad \delta \pi^{a}=\delta \Lambda_{b}^{a} \pi^{b},
$$

with arbitrary functions $\delta \Lambda_{b}^{a}(\Gamma)$, one generates the following effective changes in the integrand of $(2.5)$ :

$$
\begin{gathered}
\delta G_{a}=\left(G_{a}, \delta X\right)+G_{b} \delta \Lambda_{a}^{b}, \\
\delta H=-\Delta \delta X+(H, \delta X)-(-1)^{\varepsilon_{a}} \delta \Lambda_{a}^{a},
\end{gathered}
$$

where the first and second terms in the r.h.s. of (2.15) describe, respectively, the most general changes of the hipergauge surface and its basis.

On the other hand, the transformations (2.15) retain the form of the equations (2.8), (2.9) by inducing the following variations of structure coefficients :

$$
\delta U_{b c}^{a}=\left(U_{b c}^{a}, \delta X\right)+\left[\left(\delta \Lambda_{b}^{a}, G_{c}\right)+U_{b d}^{a} \delta \Lambda_{c}^{d}\right]-\left[\left(\delta \Lambda_{c}^{a}, G_{b}\right)+U_{c d}^{a} \delta \Lambda_{b}^{d}\right](-1)^{\left(\varepsilon_{b}+1\right)\left(\varepsilon_{c}+1\right)}-\delta \Lambda_{d}^{a} U_{b c}^{d},
$$




$$
\delta V_{b}^{a}=\left(V_{b}^{a}, \delta X\right)+V_{d}^{a} \delta \Lambda_{b}^{d}-\delta \Lambda_{d}^{a} V_{b}^{d}+\left[\Delta \delta \Lambda_{b}^{a}-\left(H, \delta \Lambda_{b}^{a}\right)\right](-1)^{\varepsilon_{a}} .
$$

Due to the arguments analogous to the ones given in Refs. [1,2] we conclude that the modified functional integral (2.5) does not depend on hypergauge fixing.

\section{A Modified Version to the $n$-th Level Formalism}

In this Section we construct inductively the $n$-th level modified functional integral for $n=$ $2,3, \ldots$

First, let us define recursively the $n$-th level set of variables of the field-antifield phase space

$$
\Gamma^{(n) A_{(n)}} \equiv\left\{\Gamma^{(n-1) A_{(n-1)}} ; \pi^{(n-1) a}, \pi_{a}^{*(n-1)}\right\}
$$

where

$$
\Gamma^{(1) A_{(1)}} \equiv \Gamma^{A}, \quad \pi^{(1) a} \equiv \pi^{a} .
$$

with $\pi^{(n-1) a}$ and $\pi_{a}^{*(n-1)}$ to be the $(n-1)$-th level Lagrangian multipliers and their conjugated antifields, respectively, so that

$$
\varepsilon\left(\pi^{(n) a}\right)=\varepsilon\left(\pi_{a}^{*(n)}\right)+1=\varepsilon_{a}+n-1 .
$$

In what follows all the antibrackets, $($,$) , are understood to include the totally extended$ set (3.2) of field-antifield variables, and the only nonzero elementary antibrackets for the Lagrangian multipliers are

$$
\left(\pi^{(n) a}, \pi_{b}^{(n)}\right)=\delta^{(m)(n)} \delta_{b}^{a} .
$$

Further, one constructs recursively the nilpotent operators $\Delta^{(n)}$ :

$$
\begin{gathered}
\Delta^{(n)} \equiv \Delta^{(n-1)}+(-1)^{\left(\varepsilon_{a}+n\right)} \frac{\partial}{\partial \pi^{(n-1) a}} \frac{\partial}{\partial \pi_{a}^{*(n-1)}}, \\
\Delta^{(1)} \equiv \Delta .
\end{gathered}
$$

Let us assign to the $n$-th level, $n \geq 2$, the corresponding Planck constant $\hbar^{(n)}, \varepsilon\left(\hbar^{(n)}\right)=0$, in addition to the usual one $\hbar$, together with the new quantum number called the Planck parity $\mathrm{Pl}^{(n)}$ :

$$
\mathrm{Pl}^{(n)}\left(\Gamma^{(n-1)}\right)=\mathrm{Pl}^{(n)}(\hbar)=0
$$




$$
\mathrm{Pl}^{(n)}\left(\hbar^{(n)}\right)=\mathrm{Pl}^{(n)}\left(\pi^{(n-1)}\right)=-\mathrm{Pl}^{(n)}\left(\pi^{*(n-1)}\right)=1 .
$$

The $n$-th level quantum action $W^{(n)}\left(\Gamma^{(n)} ; \hbar ; \hbar^{(n)}\right)$ is defined to satisfy the quantum master equation:

$$
\Delta^{(n)} \exp \left\{\frac{\imath}{\hbar^{(n)}} W^{(n)}\left(\Gamma^{(n)} ; \hbar ; \hbar^{(n)}\right)\right\}=0
$$

The action $W^{(n)}$ possesses the quantum numbers:

$$
\varepsilon\left(W^{(n)}\left(\Gamma^{(n)} ; \hbar ; \hbar^{(n)}\right)\right)=0, \quad \mathrm{Pl}^{(n)}\left(W^{(n)}\left(\Gamma^{(n)} ; \hbar ; \hbar^{(n)}\right)\right)=1
$$

and has the following series expansion in powers of $\hbar^{(n)}, \pi^{(n-1)}, \pi^{*(n-1)}$ :

$$
\begin{gathered}
W^{(n)}\left(\Gamma^{(n)} ; \hbar ; \hbar^{(n)}\right)=\Omega^{(n)}\left(\Gamma^{(n)} ; \hbar\right)+\imath \hbar^{(n)} \Xi^{(n)}\left(\Gamma^{(n)} ; \hbar\right)+\left(\imath \hbar^{(n)}\right)^{2} \tilde{\Omega}^{(n)}\left(\Gamma^{(n)} ; \hbar\right)+\ldots \\
\Omega^{(n)}\left(\Gamma^{(n)} ; \hbar\right)=G_{a}^{(n-1)}\left(\Gamma^{(n-1)} ; \hbar\right) \pi^{(n-1) a}+ \\
+\frac{1}{2} \pi_{c}^{*(n-1)} U_{a b}^{(n-1) c}\left(\Gamma^{(n-1)} ; \hbar\right) \pi^{(n-1) b} \pi^{(n-1) a}(-1)^{\left(\varepsilon_{a}+n\right)}+\ldots, \\
\Xi^{(n)}\left(\Gamma^{(n)} ; \hbar\right)=H^{(n-1)}\left(\Gamma^{(n-1)} ; \hbar\right)+\pi_{a}^{*(n-1)} V_{b}^{(n-1) a}\left(\Gamma^{(n-1)} ; \hbar\right) \pi^{(n-1) b}+\ldots, \\
\tilde{\Omega}^{(n)}\left(\Gamma^{(n)} ; \hbar\right)=\pi_{a}^{*(n-1)} \tilde{G}^{(n-1) a}\left(\Gamma^{(n-1)} ; \hbar\right)+\ldots
\end{gathered}
$$

Substituting the expansion (3.11) for $W^{(n)}$ into the quantum master equation (3.9), we find the following equations for the functions $\Omega^{(n)}, \Xi^{(n)}, \tilde{\Omega}^{(n)}, n \geq 2$ :

$$
\begin{gathered}
\left(\Omega^{(n)}, \Omega^{(n)}\right)=0, \\
\left(\Omega^{(n)}, \Xi^{(n)}\right)=\Delta^{(n)} \Omega^{(n)}, \\
\left(\Omega^{(n)}, \tilde{\Omega}^{(n)}\right)=\Delta^{(n)} \Xi^{(n)}-\frac{1}{2}\left(\Xi^{(n)}, \Xi^{(n)}\right) .
\end{gathered}
$$

To the lowest orders in $\pi^{(n-1)}, \pi^{*(n-1)}$ these equations give:

$$
\begin{gathered}
\left(G_{a}^{(n-1)}, G_{b}^{(n-1)}\right)=G_{c}^{(n-1)} U_{a b}^{(n-1) c}, \\
\left(H^{(n-1)}, G_{a}^{(n-1)}\right)=\Delta^{(n-1)} G_{a}^{(n-1)}+U_{b a}^{(n-1) b}(-1)^{\left(\varepsilon_{b}+n-1\right)}-G_{b}^{(n-1)} V_{a}^{(n-1) b},
\end{gathered}
$$




$$
\Delta^{(n-1)} H^{(n-1)}-\frac{1}{2}\left(H^{(n-1)}, H^{(n-1)}\right)+V_{a}^{(n-1) a}=G_{a}^{(n-1)} \tilde{G}^{(n-1) a},
$$

The general involution relations (3.18) and usual gauge admissibility conditions are the only restrictions imposed on the hypergauge functions $G_{a}^{(n-1)}\left(\Gamma^{(n-1)} ; \hbar\right)$.

At $n=2$ we identify in (3.18) - (3.20) :

$$
\begin{gathered}
G_{a}^{(1)} \equiv G_{a}, \quad U_{a b}^{(1) c} \equiv U_{a b}^{c}, \\
H^{(1)} \equiv H, \quad V_{a}^{(1) b} \equiv V_{a}^{b}, \quad \tilde{G}^{(1) a} \equiv \tilde{G}^{a} .
\end{gathered}
$$

At $n>2$ the equation (3.20) is certainly compatible with the one (3.19) but already does not follow from the latter. We consider the equations (3.19), (3.20) at $n>2$ to determine $H^{(n-1)}$ under the boundary conditions

$$
\left.H^{(n-1)}(\Gamma ; \hbar)\right|_{G^{(n-1)}=0, \pi^{(n-2)}=0}=-\frac{\imath}{\hbar} W^{(n-2)}\left(\Gamma^{(n-2)} ; \hbar ; \hbar\right)-\ln \operatorname{Ber}\left(\pi^{(n-2)}, G^{(n-1)}\left(\Gamma^{(n-2)} ; \hbar\right)\right),
$$

where we identify at $n=3$ :

$$
W^{(1)}\left(\Gamma^{(1)} ; \hbar ; \hbar\right) \equiv W(\Gamma ; \hbar) .
$$

The $n$-th level modified functional integral is defined to be:

$$
Z^{(n)}=\int \exp \left\{\frac{\imath}{\hbar}\left[W^{(n)}\left(\Gamma^{(n)} ; \hbar ; \hbar\right)+G_{a}^{(n)}\left(\Gamma^{(n)} ; \hbar\right) \pi^{(n) a}\right]-H^{(n)}\left(\Gamma^{(n)} ; \hbar\right)\right\} d \mu^{(n)},
$$

where the action $W^{(n)}\left(\Gamma^{(n)} ; \hbar ; \hbar^{(n)}\right)$ was defined above, the final functions $G_{a}^{(n)}\left(\Gamma^{(n)} ; \hbar\right)$ and $H^{(n)}\left(\Gamma^{(n)} ; \hbar\right)$ are subordinated by hand to satisfy the equations obtained from the ones (3.18) - (3.20), (3.23) by making formal replacement $n-1 \rightarrow n$, the measure $d \mu^{(n)}$ is defined recursively as follows :

$$
\begin{gathered}
d \mu^{(n)}=d \mu^{(n-1)} d \pi^{*(n-1)} d \pi^{(n)}, \quad n \geq 2, \\
d \mu^{(1)} \equiv d \mu .
\end{gathered}
$$

By making use of the transformations

$$
\delta \Gamma^{(n) A_{(n)}}=\left(\Gamma^{(n) A_{(n)}},-W^{(n)}+G_{a}^{(n)} \pi^{(n) a}+\imath \hbar H^{(n)}\right) \frac{\imath}{2 \hbar} \delta X^{(n)}+\frac{1}{2}\left(\Gamma^{(n) A_{(n)}}, \delta X^{(n)}\right),
$$




$$
\delta \pi^{(n) a}=\left[U_{b c}^{(n) a} \pi^{(n) c} \pi^{(n) b}(-1)^{\left(\varepsilon_{b}+n\right)}+2 \imath \hbar V_{b}^{(n) a} \pi^{(n) b}+2(\imath \hbar)^{2} \tilde{G}^{(n) a}\right] \frac{\imath}{2 \hbar} \delta X^{(n)}+\delta \Lambda_{b}^{(n) a} \pi^{(n) b},
$$

where $\delta X^{(n)}$ is an arbitrary Fermion function, we induce the following variations in the integrand of (3.25) :

$$
\begin{gathered}
\delta G_{a}^{(n)}=\left(G_{a}^{(n)}, \delta X^{(n)}\right)+G_{b}^{(n)} \delta \Lambda_{a}^{(n) b}, \\
\delta H^{(n)}=-\Delta^{(n)} \delta X^{(n)}+\left(H^{(n)}, \delta X^{(n)}\right)+(-1)^{\left(\varepsilon_{a}+n\right)} \delta \Lambda_{a}^{(n) a}
\end{gathered}
$$

The equations for $H^{(n)}$ retain their form under the variations (3.30), (3.31) by inducing the following transformations for structure coefficients :

$$
\begin{gathered}
\delta U_{b c}^{(n) a}=\left(U_{b c}^{(n) a}, \delta X^{(n)}\right)+\left[\left(\delta \Lambda_{b}^{(n) a}, G_{c}^{(n)}\right)+U_{b d}^{(n) a} \delta \Lambda_{c}^{(n) d}\right]-\left[\left(\delta \Lambda_{c}^{(n) a}, G_{b}^{(n)}\right)+\right. \\
\left.+U_{c d}^{(n) a} \delta \Lambda_{b}^{(n) d}\right](-1)^{\left(\varepsilon_{b}+n\right)\left(\varepsilon_{c}+n\right)}-\delta \Lambda_{d}^{(n) a} U_{b c}^{(n) d} \\
\delta V_{b}^{(n) a}=\left(V_{b}^{(n) a}, \delta X^{(n)}\right)+V_{d}^{(n) a} \delta \Lambda_{b}^{(n) d}-\delta \Lambda_{d}^{(n) a} V_{b}^{(n) d}+\left[\Delta \delta \Lambda_{b}^{(n) a}+\left(H^{(n)}, \delta \Lambda_{b}^{(n) a}\right)\right](-1)^{\left(\varepsilon_{a}+n\right)} \\
\delta \tilde{G}^{(n) a}=\left(\tilde{G}^{(n) a}, \delta X^{(n)}\right)-\delta \Lambda_{b}^{(n) a} \tilde{G}^{(n) a} .
\end{gathered}
$$

By the same reasoning as the one applied to (2.5), we conclude that the functional integral (3.25) does not depend on the final hypergauge functions $G_{a}^{(n)}$. Choosing then $G_{a}^{(n)}$ to take the simplest form :

$$
G_{a}^{(n)}=\pi_{a}^{*(n-1)},
$$

we obtain

$$
Z^{(n)}=Z^{(n-1)}, \quad n \geq 2,
$$

and thus arrive at the final reduction formula

$$
Z^{(n)}=Z^{(1)} \equiv Z
$$




\section{Conclusion}

So, we have constructed the multilevel field-antifield formalism with the most general Lagrangian hypergauges.

The characteristic feature of the new generalization suggested is the appearance of extra measure factors in the corresponding functional integrals. It is established that these extra factors can be included naturally into the multilevel scheme by modifying the boundary conditions to the quantum master equation.

At the first level it appears to be just possible to construct the extra measure factor $\exp (-H)$ in an explicitly covariant form [6], that is

$$
\exp (-H)=\left[J M^{-1} \operatorname{Ber}(F, G)\right]^{1 / 2},
$$

where $G_{a}$ satisfy $(2.8), F^{a}, a=1, \ldots, N, \varepsilon\left(F^{a}\right)=\varepsilon_{a}+1$, are some functions such that the replacement $\Gamma^{A} \rightarrow \bar{\Gamma}^{A} \equiv\left\{F^{a} ; G_{a}\right\}$ is an invertible reparametrization whose Jacobian is denoted by $J$,

$$
J \equiv \operatorname{Ber}\left(\frac{\partial \bar{\Gamma}}{\partial \Gamma}\right)
$$

It is an important property that the expression (4.1), being taken on the hypergauge surface, does not depend on $F^{a}$. One can also show that this expression represents the general solution to the equation (2.9).

Substituting (4.1) into (2.5) we obtain the following representation for the first level functional integral :

$$
Z=\int \exp \left(\frac{i}{\hbar} W\right) \delta(G)[J M \operatorname{Ber}(F, G)]^{1 / 2} d \Gamma .
$$

Due to the presence of the square root, [ $]^{1 / 2}$, complete measure factor, entering the integrand of (4.3), cannot be, in general, parametrized by means of the integral over new fields with a local action.

As for the higher level case, the situation seems to be more complicated. Regrettably, we are unable, for the present, to construct the corresponding extra measure factors $\exp \left(-H^{(n)}\right)$, $n \geq 2$, in an explicity covariant closed form.

Acknowledgement. We are thankful to Dr. O.M.Khudaverdian for informing us on the representation (4.1) prior to its publication. 


\section{References}

[1] I.A.Batalin and I.V.Tyutin, Int. J. Mod. Phys. A8 2333 (1993).

[2] I.A.Batalin and I.V.Tyutin, Mod. Phys. Lett. A8 3673 (1993).

[3] I.A.Batalin and G.A.Vilkovisky, Phys. Lett. B102 27 (1981).

[4] I.A.Batalin and G.A.Vilkovisky, Phys. Rev. D28 2567 (1983).

[5] For a review see: M.Henneaux, Nucl. Phys. B (Proc. Suppl.) 18A 47 (1990).

[6] O.M.Khudaverdian, private communication. 